D

Von Christoph Bals onshandelssystem vorgelegt, der es in sich hat (vgl. auch Ökologisches Wirtschaften 6/01, S. 2): Absolute Reduktionsziele für die großen Emittenten von Kohlendioxid; die Notwendigkeit, entweder das eigene Ziel zu erreichen, oder überzählige Emissionszertifikate von anderen Akteuren in der EU zu kaufen; saftige Sanktionen, wenn die Ziele verfehlt werden.

Einige Akteure der Wirtschaft scheuen den rauen Wind des Marktes und würden lieber am gemütlichen Nest der ,freiwilligen Selbstverpflichtung“ festhalten. Angetrieben von der BASF bläst der Verband der Chemischen Industrie ins Protesthorn. Der Emissionshandel mit seiner harten Begrenzung des Emissionsausstoßes für einzelne Unternehmen passt nicht zum rheinischBerliner Politikstil des Klüngels. Plötzlich werden harte Entscheidungen der Zuteilung, wirkliche Transparenz beim Monitoring und bei der Verifizierung sowie ein hartes Sanktionssystem notwendig. Ein funktionierender Markt braucht einen Ordnungsrahmen, dann hat er viele Vorteile:

- Ein mengenorientiertes System wie das von der Kommission konzipierte Cap and Trade- System garantiert wie kein anderes Konzept $\ddot{o} k o$ logische Treffsicherheit. Zugleich aber signalisiert es der Wirtschaft: Wir sind nicht daran interessiert, euch im Detail zu regulieren. Wie ihr das umsetzt, ist eure Sache. Und auch, wo - solange die Umweltintegrität gewahrt bleibt.

- Ein solches System ermöglicht, ökonomische Chancen zu nutzen und unternehmerische $R i$ siken erfolgreich zu managen. Beim Klimagipfel in Marrakesch machten institutionelle Anleger deutlich, dass sie von Unternehmen mit hohen Kohlenstoffrisiken nun ein geeignetes Risikomanagement-System gegenüber dem regulativen Risiko weiterer politischer Regulierungen erwarten. Wo dies nicht durch Diversifikation des Portfolios in Richtung erneuerbare Energieträger und Energieeffizienz geschieht, dürfte der Optionen-Handel im Rahmen eines Emissionshandelssystems die passende Möglichkeit sein.

- Dieses Instrument gestattet Unternehmen die Flexibilität, beim Erreichen der Klimaschutzziele neue klimafreundliche Investitionen erst dann zu tätigen, wenn die alten Investitionen abgeschrieben sind. Indem Kapitalvernichtung damit so weit wie möglich vermieden wird -

\section{Einstieg in ernsthaften Klimaschutz?}

\section{Mit dem geplanten europäischen} Emissionshandel steht der Einstieg in ernsthaften Klimaschutz vor der Tür. Auch in Deutschland wird im Rahmen einer Arbeitsgruppe über die Umsetzung diskutiert. Aufgrund des Widerstands einiger Akteure der Wirtschaft drohte diese zu scheitern. Die verbliebenen Akteure sollten die Chance für ein ökologisch integres Modell nutzen.

ohne die Umweltziele zu gefährden - werden auch negative Arbeitsmarkteffekte eingeschränkt; durch den Innovationsanreiz sind zugleich positive Jobeffekte zu erwarten.

- Es ist ein nicht zu unterschätzender Strukturwandel innerhalb der betroffenen Unternehmen zu erwarten. Nicht mehr alleine die Umweltleute, sondern auch die Finanzabteilung hat dann ein Interesse, dass ein intensiver Suchprozess zur Klimagasreduzierung beginnt.

- Ein internationales Emissionshandelsregime in Europa wird der wohl stärkste Anziehungsfaktor für die USA, sich den internationalen Klimaschutzbemühungen über kurz oder lang anzuschließen. Der Druck der transnationalen Unternehmen in diese Richtung wächst bereits.

- Aus ökologischer Perspektive, aber vor allem auch wegen der notwendigen Liquidität des Marktes, ist es sinnvoll, dass die EU die Initiative ergriff, bevor ein Flickenteppich nationaler, nicht kompatibler Märkte entsteht. Bereits die beiden ersten nationalen Konzepte, Großbritannien und Dänemark, sind nicht kompatibel.

\section{Große Chancen mit Risiken}

Der Emissionshandel auf Unternehmensebene kann ein sehr zielgenaues und wirkungsmächtiges Instrument sein. Viele Akteure, die am Klimaschutz keinerlei Interesse haben, betreten das Spielfeld. Das ist gewünscht - der Klimaschutz darf nicht eine Spielwiese für Idea- listen bleiben. Aber es macht auch deutlich: $\mathrm{Ab}$ jetzt muss es strikte Regeln geben, die dafür sorgen, dass tatsächlich Emissionen und nicht $\mathrm{Pa}$ pierbuchungen gehandelt werden; dass tatsächlich die Innovationen angestoßen werden, die wir brauchen. Wichtigen Risiken für die Integrität des Systems gilt es vorzubeugen:

- Die EU-Richtlinie sieht vor, dass zu einem späteren Zeitpunkt zusätzlich auch projektbasierter Handel in das System einbezogen werden soll. Dies ist im Prinzip zu begrüßen. Aber die Spielregeln von Kyoto reichen leider nicht aus, die ökologische und soziale Integrität dieser Projekte sicherzustellen. Hier ist die EU in der Pflicht, diesbezüglich einen ,goldenen Standard" zu erarbeiten.

- Einige Länder - vor allem Russland - haben in Kyoto Ziele erhalten, die sie selbst bei starkem Wirtschaftswachstum deutlich unterschreiten. Der EU-Handel zwischen Unternehmen muss, wie von der Kommission vorgesehen, gegenüber dieser "beißen Luft" abgedichtet bleiben.

- Der Verzicht auf Ökosteuer für die am Emissionshandel teilnehmenden Unternehmen ist kein Tabu. Hingegen wäre es sinnvoll, für die nicht teilnehmenden Unternehmen den vollen Satz einzuführen. Unverzichtbar aber bleibt als zusätzliche Maßnahme die beschleunigte Markteinführung erneuerbarer Energien und anderer innovativer Technologien wie Brennstoffzellen, die langfristig die Staffel des Klimaschutzes übernehmen können

Ende des Jahres wollten einige Wirtschaftsakteure der Bundesregierung, den Umweltverbänden und den Kollegen aus der Wirtschaft als Voraussetzung für eine gemeinsame Stellungnahme ein „Nein“ zum EU-Richtlinienvorschlag abtrotzen. Sie brachten die Arbeitsgruppe an den Rand des Scheiterns. In wesentlich kleineren, thematisch aufgespaltenen Arbeitsgruppen gibt es nun einen neuen Versuch, zu Kompromissen zu kommen. Dies kann nur gelingen, wenn dabei nicht die Klimaschutzziele und die Ernsthaftigkeit des politischen Willens, diese zu erreichen, direkt oder indirekt in Frage gestellt werden.

\section{Der Autor}

Christoph Bals ist für die Nord-Süd NGO Germanwatch Mitglied der Arbeitsgruppe Emissionshandel. Kontakt: Germanwatch e.V., Kaiserstr. 201 , 53113 Bonn. Tel. 0228/ 60492-0, Fax -19, E-mail: bals@germanwatch.org 
(c) 20I0 Authors; licensee IÖW and oekom verlag. This is an article distributed under the terms of the Creative Commons Attribution Non-Commercial No Derivates License (http://creativecommons.org/licenses/by-nc-nd/3.o/), which permits unrestricted use, distribution, and reproduction in any medium, provided the original work is properly cited. 